\title{
Prostate Cancer Grade and Stage Misclassification in Active Surveillance Candidates: Black Versus White Patients
}

\author{
Lara Franziska Stolzenbach, MD,2; Giuseppe Rosiello, MD ${ }^{1,3}$; Angela Pecoraro, MD ${ }^{1,4}$; Carlotta Palumbo, MD ${ }^{1,5}$; \\ Stefano Luzzago, MD ${ }^{1,6}$; Marina Deuker, MD ${ }^{1,7}$; Zhe Tian, MD'; Anne-Sophie Knipper, MD²; Raisa Pompe, MD ${ }^{8}$; \\ Kevin C. Zorn, MD'; Shahrokh F. Shariat, MD9,10; Felix K.H. Chun, MD; Markus Graefen, MD²; \\ Fred Saad, $\mathrm{MD}^{1}$; and Pierre I. Karakiewicz, $\mathrm{MD}^{1}$
}

\begin{abstract}
Background: Misclassification rates defined as upgrading, upstaging, and upgrading and/or upstaging have not been tested in contemporary Black patients relative to White patients who fulfilled criteria for very-low-risk, low-risk, or favorable intermediate-risk prostate cancer. This study aimed to address this void. Methods: Within the SEER database (2010-2015), we focused on patients with very low, low, and favorable intermediate risk for prostate cancer who underwent radical prostatectomy and had available stage and grade information. Descriptive analyses, temporal trend analyses, and multivariate logistic regression analyses were used. Results: Overall, 4,704 patients with very low risk (701 Black vs 4,003 White), 17,785 with low risk (2,696 Black vs 15,089 White), and 11,040 with favorable intermediate risk (1,693 Black vs 9,347 White) were identified. Rates of upgrading and/or upstaging in Black versus White patients were respectively $42.1 \%$ versus $37.7 \%$ (absolute $\Delta=+4.4 \% ; P<.001$ ) in those with very low risk, $48.6 \%$ versus $46.0 \%$ (absolute $\Delta=+2.6 \% ; P<.001$ ) in those with low risk, and $33.8 \%$ versus $35.3 \%$ (absolute $\Delta=-1.5 \%$; $P=.05)$ in those with favorable intermediate risk. Conclusions: Rates of misclassification were particularly elevated in patients with very low risk and low risk, regardless of race, and ranged from $33.8 \%$ to $48.6 \%$. Recalibration of very-low-, low-, and, to a lesser extent, favorable intermediate-risk active surveillance criteria may be required. Finally, our data indicate that Black patients may be given the same consideration as White patients when active surveillance is an option. However, further validations should ideally follow.
\end{abstract}

J Natl Compr Canc Netw 2020;18(11):1492-1499 doi: $10.6004 /$ jnccn.2020.7580

\footnotetext{
${ }^{1}$ Cancer Prognostics and Health Outcomes Unit, Division of Urology, University of Montreal Health Center, Montreal, Quebec, Canada; ${ }^{2}$ Martini-Klinik Prostate Cancer Center, University Hospital Hamburg-Eppendorf, Hamburg, Germany; ${ }^{3}$ Department of Urology and Division of Experimental Oncology, Urological Research Institute, IRCCS San Raffaele Scientific Institute, Milan, Italy;

${ }^{4}$ Department of Urology, San Luigi Gonzaga Hospital, University of Turin, Turin, Italy; ${ }^{5}$ Urology Unit, ASST Spedali Civili of Brescia, Department of Medical and Surgical Specialties, Radiological Science and Public Health, University of Brescia, Brescia, Italy; ${ }^{6}$ Department of Urology, European Institute of Oncology, IRCCS, Milan, Italy; ${ }^{7}$ Department of Urology, University Hospital Frankfurt, Frankfurt am Main, Germany; ${ }^{8}$ Department of Urology, Medical University of Hamburg, Hamburg, Germany; ${ }^{9}$ Department of Urology, Comprehensive Cancer Center, Medical University of Vienna, Vienna, Austria; and ${ }^{10}$ Institute of Urology and Reproductive Health, I.M. Sechenov First Moscow State Medical University, Moscow, Russia.
}

\section{Background}

The NCCN Clinical Practice Guidelines in Oncology (NCCN Guidelines) for Prostate Cancer (PCa) support active surveillance (AS) in patients at very low risk, low risk, and even favorable intermediate risk for PCa. ${ }^{1}$ These definitions originate from studies that relied almost exclusively on White patients. For example, Epstein et $\mathrm{al}^{2}$ and Bastian et $\mathrm{al}^{3}$ validated the low-risk criteria in 157 and 237 patients, respectively, which included Black patients but did not stratify by race. Consequently, largescale, population-based validation studies of these criteria are needed in racial groups other than White patients.

Our study addressed this void and tested for AS misclassification rates in Black patients who fulfilled at least 1 of the AS criteria but underwent radical prostatectomy (RP) with available pathologic staging. Rates were then compared with those for White patients. We hypothesized that equally low misclassification applies to both races.

\section{Methods}

\section{Study Population}

Data were obtained from the SEER database for the period 2010 through $2015 .{ }^{4}$ We focused on patients with histologically confirmed nonmetastatic adenocarcinoma of the prostate (ICD-O C61.9) who had available prostatespecific antigen (PSA) values and were treated using RP. Patients were categorized according to the AS criteria into 1 of 3 groups: very low, low, or favorable intermediate risk. ${ }^{1}$ The very-low-risk criteria required PSA level $<10 \mathrm{ng} / \mathrm{mL}$, Gleason grade group (GGG) 1 , cT stage 1 , and $<3$ positive prostate biopsy cores with $<50 \%$ cancer per core. Low-risk PCa criteria required PSA level $<10 \mathrm{ng} / \mathrm{mL}$, GGG 1, and cT1-2a. Favorable intermediate-risk PCa criteria required

See JNCCN.org for supplemental online content. 
GGG $\leq 2,<50 \%$ positive biopsy cores, and 1 intermediate risk factor, including cT2b/c or GGG 3 or PSA level of $10-20 \mathrm{ng} / \mathrm{mL} .{ }^{1}$ Patients with an unknown GGG $(\mathrm{n}=2,893)$, an unknown cT stage $(n=11,529)$, or an unknown number of positive and negative biopsy cores $(n=49,969)$ were excluded. These selection criteria yielded 28,923 patients.

\section{Variable Definition}

Race was defined as Black or White. Covariables consisted of age at diagnosis, year of diagnosis, preoperative PSA level, total number of biopsy cores, number of positive biopsy cores, cT stage (cT1c, cT2a, cT2b, cT2c, cT2), and percentage of positive biopsy cores (core ratio), which was calculated by dividing the number of positive cores by the total number of cores (first quartile, $<0.17$; second quartile, 0.17 to $<0.33$; third quartile, 0.33 to $<0.50$; fourth quartile, $\geq 0.50$ ).

\section{Statistical Analyses}

Descriptive statistics focused on frequencies and proportions for categorical variables. Means, medians, and interquartile ranges were reported for continuously coded variables. Chi-square testing was used for the statistical significance in proportion differences. The $t$ test and Kruskal-Wallis test examined the statistical significance of differences in means and medians. The rates of upgrading, defined as an increase of at least 1 GGG from the prostate biopsy GGG to the GGG at final pathology at RP; the rates of upstaging, defined as a non-organconfined tumor (stage $\mathrm{pT} 3+/ \mathrm{pN1}$ ) at $\mathrm{RP}$; and the rates of upgrading and/or upstaging, defined as a combination of both, were tabulated for Black and White patients. Specifically, 3 particular endpoints were examined: rates of upgrading, rates of upstaging, and rates of combined upgrading and/or upstaging. The 3 endpoints were stratified according to Black versus White race. Additional stratifications of each endpoint were performed; in particular, rates of combined upgrading and/or upstaging were specified for upgrading to GGG 3, 4, or 5 and/or upstaging to pT3+,pN1. Subsequently, multivariate logistic regression (MLR) models tested the effect of Black race on upgrading, upstaging, and upgrading and/or upstaging (see supplemental eTables 1-3, available with this article at JNCCN.org). Finally, temporal trends for upgrading but not upstaging were examined due to very low observation counts. Statistical analyses were performed using $\mathrm{R}$ version 3.4.1 (R Foundation for Statistical Computing). All tests were 2 -sided with a level of significance set at $P<.05$.

\section{Results}

\section{Descriptive Characteristics}

Of all 210,100 patients treated with RP, 4,704 (2.2\%), 17,785 (8.4\%), and 11,040 (5.2\%) fulfilled the very-low-risk, low-risk, and favorable intermediate-risk AS criteria, respectively (Table 1$)$. Of those, $14.9 \%(\mathrm{n}=701 ; P<.001), 15.1 \%$ $(\mathrm{n}=2,696 ; P<.001)$, and $15.3 \%(\mathrm{n}=1,693 ; P<.001)$, respectively, were Black. Overall, Black patients were younger than White patients $(P<.001)$. No clinically meaningful or statistically significant differences in tumor characteristics were recorded in Black patients compared with White patients (Table 1).

In patients with very low risk (Figure 1), Black patients had higher rates of upgrading $(40.1 \%$ vs $36.3 \%$; absolute $\Delta=+3.8 \% ; P<.001)$, upstaging $(10.1 \%$ vs $6.9 \%$; absolute $\Delta=+3.2 \% ; P=.01$ ), and upgrading and/or upstaging $(42.1 \%$ vs $37.7 \%$; absolute $\Delta=+4.4 \%$; $P<.001)$. Similarly, as shown in Table 2 , the rates of upgrading to GGG 3 , 4, or 5 and/or upstaging were higher in Black versus White patients $(8.9 \%$ vs $7.3 \%$; $P<.01)$. Upgrading rates into each GGG were as follows: GGG 2, $33.1 \%$ versus $30.3 \%$; GGG $3,5.4 \%$ versus $4.6 \%$; GGG 4 , $1.3 \%$ versus $0.9 \%$; and GGG $5,0.3 \%$ versus $0.6 \%$ in Black versus White patients, respectively (all $P>.05$ ). In the subgroup upgraded to GGG 2, Black patients exhibited a higher proportion of upgrading with concomitant upstaging than White patients $(18.2 \%$ vs $13.4 \%$; $P<.01$ ). Similarly, a higher proportion of upgrading with upstaging was also noted in those Black patients upgraded to GGG 3 , 4 , or 5 ( $30.6 \%$ vs $25.9 \%$; $P<.01$ ). Finally, in upstaged patients, no statistically significant differences were recorded between Black and White patients $(7.9 \%$ vs $4.6 \% ; P=.2)$. In MLR models, Black race independently predicted higher rates of upgrading (odds ratio $[\mathrm{OR}], 1.23 ; 95 \% \mathrm{CI}, 1.03-1.45 ; P=.01$ ), upstaging (OR, 1.60; 95\% CI, 1.19-2.13; $P=.001)$, and upgrading and/or upstaging (OR, 1.26; 95\% CI, 1.06-1.49; $P=.007$ ) (supplemental eTable 1). Upgrading rates increased significantly over time in Black patients (39.5\% in 2010 vs $47.2 \%$ in 2015 ; estimated annual percentage change [EAPC], $4.95 \%$; $95 \% \mathrm{CI},-0.3 \%$ to $10.6 \% ; P=.01)$ and in White patients $(31.4 \%$ in 2010 vs $42.5 \%$ in 2015 ; EAPC, $5.04 \%$; 95\% CI, $3.1 \%-7.0 \%$; $P<.006$ ) (Figure 2).

In patients with low risk (Figure 1), Black patients had higher rates of upgrading $(46.0 \%$ vs $43.5 \%$; absolute $\Delta=+2.5 \% ; P<.001)$ and upgrading and/or upstaging $(48.7 \%$ vs $46.0 \%$; absolute $\Delta=+2.7 \%$; $P<.001)$, but not rates of upstaging alone $(8.8 \%$ vs $9.7 \%$; absolute $\Delta=-0.9 \% ; P<.001)$. Moreover, as shown in Table 2, no statistically significant differences in upgrading to GGG 3, 4, or 5 and/or upstaging rates were recorded between Black and White patients $(9.2 \%$ vs $8.2 \% ; P<.01)$. However, upgrading rates into each GGG were as follows: GGG 2, 39.5\% versus $37.8 \%$; GGG 3, $4.9 \%$ versus $4.4 \%$; GGG $4,1.3 \%$ versus $0.8 \%$; and GGG $5,0.3 \%$ versus $0.5 \%$ in Black versus White patients, respectively (all $P>.05$ ). In the subgroup upgraded to GGG 2 and the 
Table 1. Patient Characteristics

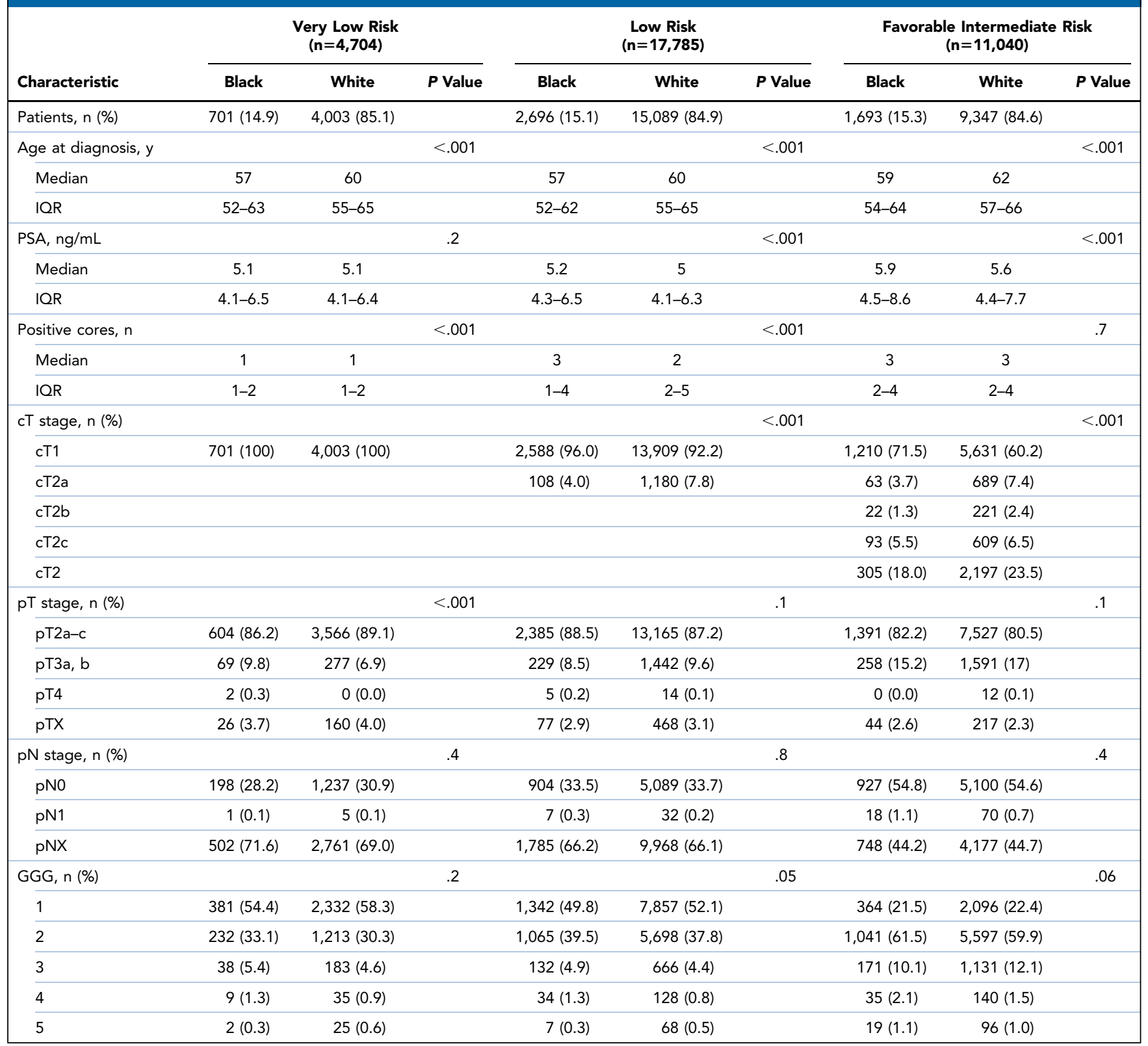

Abbreviations: cT, clinical T stage; pN, pathologic N stage; $\mathrm{PT}$, pathologic T stage; GGG, Gleason grade group; IQR, interquartile range; PSA, prostate-specific antigen.

subgroups upgraded to GGG 3 , 4, or 5 , no clinically meaningful or statistically significant differences $(P=.3)$ were recorded between Black and White patients regarding the proportions of upgrading with concomitant upstaging. Finally, in upstaged patients, no statistically significant differences were recorded between Black and White patients $(6.0 \%$ vs $7.2 \% ; P=.4)$. In MLR models, Black race independently predicted higher rates of upgrading (OR, 1.12; 95\% CI, 1.00-1.25; $P=.03$ ) and upgrading and/or upstaging (OR, 1.15; $95 \%$ CI, $1.03-1.28 ; \quad P<.01)$ but not of upstaging
(OR, 0.85; $P=.09$ ) (supplemental eTable 2). Upgrading rates increased significantly over time in Black patients $(44.3 \%$ in 2010 vs $53.0 \%$ in 2015 ; EAPC, $5.2 \%$; $95 \%$ CI, $2.1 \%-8.3 \% ; P=.02)$ and in White patients $(41.0 \%$ in 2010 vs $48.4 \%$ in 2015 ; EAPC, $3.8 \%$; $95 \%$ CI, $3.1 \%-4.5 \%$; $P<.001$ ) (Figure 2).

In patients with favorable intermediate risk, Black patients did not exhibit statistically significantly higher rates than White patients of either upgrading $(24.0 \%$ vs $24.7 \%$; absolute $\Delta=-0.7 \% ; P=.2)$, upstaging $(15.8 \%$ vs $17.4 \%$; absolute $\Delta=-1.6 \%$; $P=.06$ ), or upgrading 
A

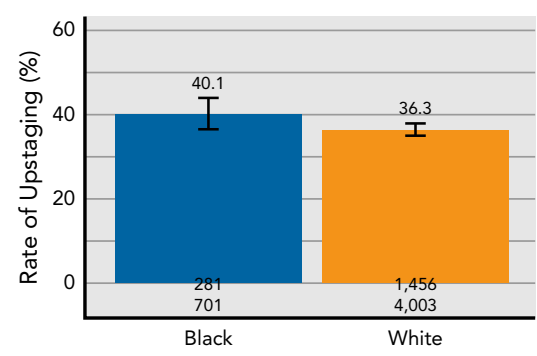

D

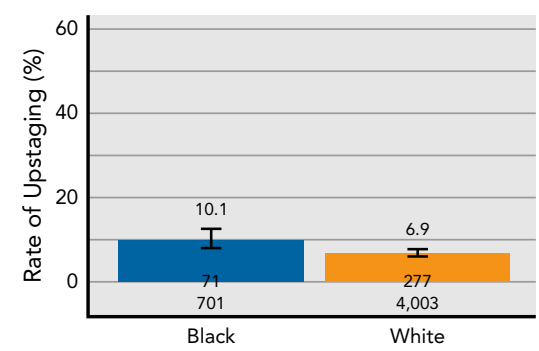

G

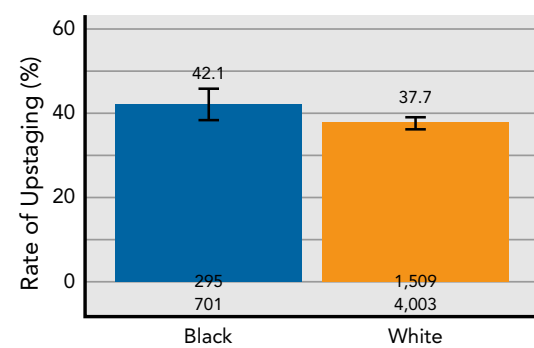

B

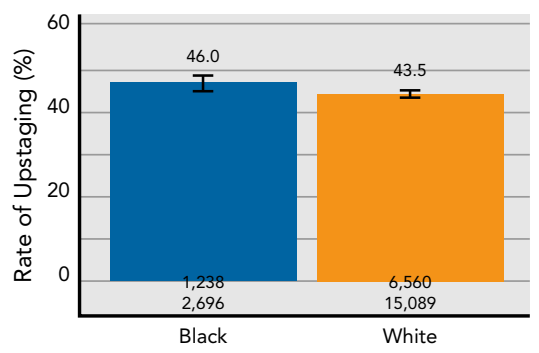

E

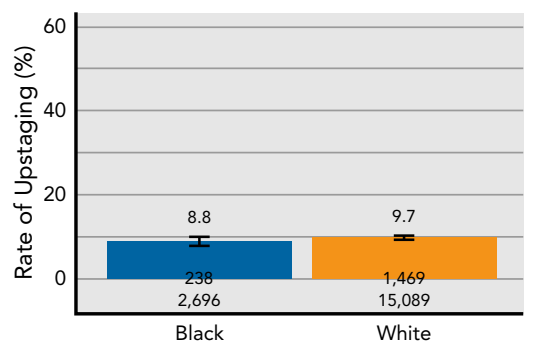

H

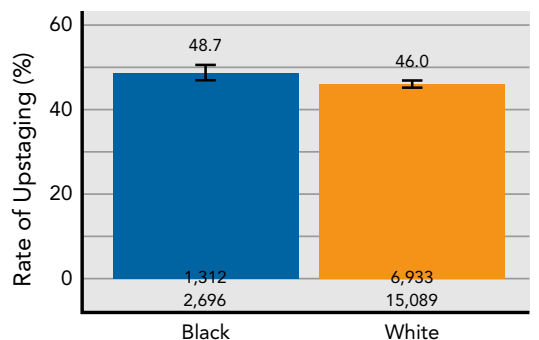

C

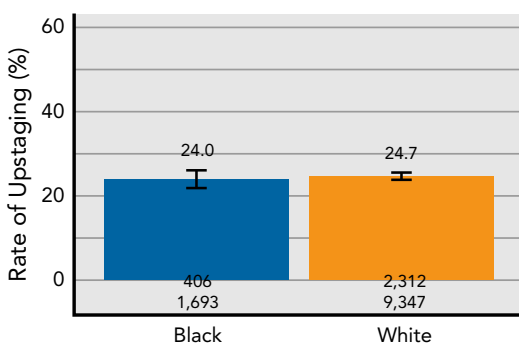

$\mathbf{F}$

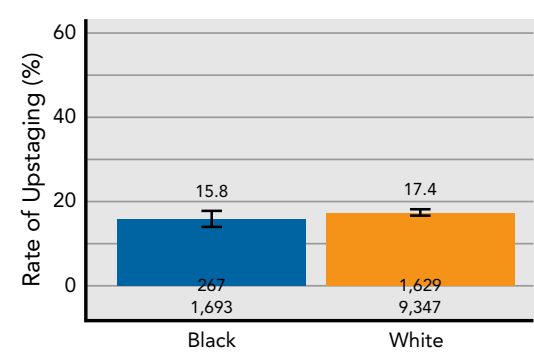

I

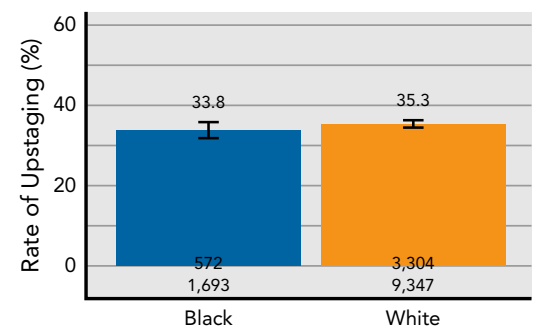

Figure 1. Barplots depicting rates of $(A-C)$ upgrading $(D-F)$ upstaging, and (G-l) upgrading and/or upstaging within the very-low risk (A, $D, G)$, low-risk $(B, E, H)$, and favorable intermediate-risk $(C, F, I)$ groups. The proportion with either upgrading, upstaging, or upgrading and/or upstaging is shown above the total population below each bar. The counts of individuals with each feature as well as the proportion between affected versus total are expressed as a percentage. Bars are accompanied by $95 \%$ confidence intervals.

and/or upstaging ( $33.8 \%$ vs $35.3 \%$; absolute $\Delta=-1.5 \%$; $P=.5$ ) (Figure 1). No statistically significant differences in upgrading to GGG 3 , 4, or 5 and/or upstaging rates were recorded between Black and White patients ( $23.1 \%$ vs $25.2 \% ; P=.2)$. Rates of upgrading into each GGG were not statistically significantly higher in Black than in White patients $(P=.05)$; subgroup analyses according to upgrading into GGG 2 or GGG 3, 4, or 5 were not performed in patients with favorable intermediate risk, because biopsy at GGG 2 was allowed. Finally, in upstaged patients, no clinically meaningful or statistically significant differences $(P=.2)$ were recorded between Black and White patients (Table 2). In MLR models, Black race failed to independently predict higher rates of upgrading, upstaging, and upgrading and/or upstaging (all $P=.05$ ) (supplemental eTable 3). Upgrading rates did not change over time in either Black (28.7\% in 2010 vs $25.3 \%$ in 2015 ; EAPC, $-4.8 \%$;
$P=.2$ ) or White patients (23.2\% in 2010 vs $25.9 \%$ in 2015 ; EAPC, $0.7 \% ; P=.7$ ) (Figure 2).

\section{Discussion}

Large-scale population-based studies that have relied on modern definitions of AS and have focused on rates of upgrading and/or upstaging in Black patients are not available. This study addressed this void. Specifically, the objective of this study was to examine upgrading, upstaging, and combined upgrading and/or upstaging rates in Black and White patients who fulfilled very-low-risk, low-risk, or favorable intermediate-risk criteria as defined in the NCCN Guidelines, ${ }^{1}$ and in whom RP pathology-derived GGG and $\mathrm{pT}$ and $\mathrm{pN}$ stages were also available. We hypothesized that upgrading, upstaging, and upgrading and/or upstaging rates do not differ between Black and White patients. Our analyses yielded several noteworthy findings. 


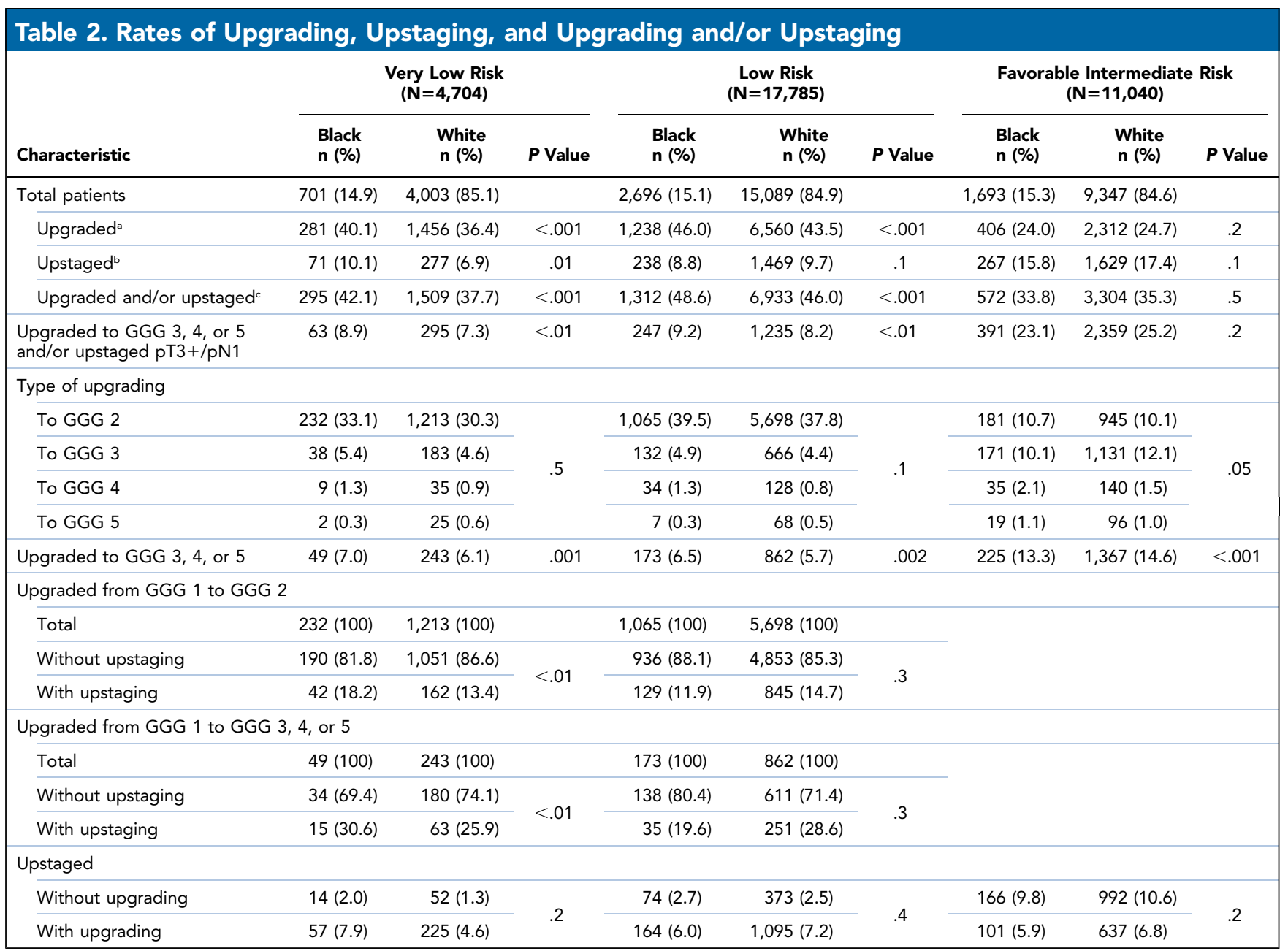

Abbreviation: GGG, Gleason grade group.

${ }^{\text {aAt }}$ least +1 GGG from prostate biopsy GGG to the GGG at the final pathology at RP.

${ }^{b}$ Non-organ-confined tumor, pT3+/pN1.

cUpstaging and/or upgrading and/or pN1.

First, we found marginal or at best very small differences in the examined endpoints between Black and White patients. The largest-magnitude differences were identified in patients with very low risk; Black patients were invariably affected by higher rates of upgrading, upstaging, or upgrading and/or upstaging, and the differences only ranged from $3.2 \%$ to $4.4 \%$. In MLR, these unadjusted rates were translated into ORs of 1.2, 1.6, and 1.3 for upgrading, upstaging, or upgrading and/or upstaging, respectively (all $P<.05$ ). However, considering the unadjusted observed rates, it is questionable whether such differences are clinically meaningful, other than to the design and planning of large-scale prospective trials that rely on the correct grade and stage assignment for randomization or stratification purposes. In patients with low risk, the differences between Black and White patients regarding upgrading, upstaging, or upgrading and/or upstaging rates were of even lesser magnitude $(-0.9 \%$ to $2.7 \%)$. In those patients, Black race reached independent predictor status only for higher upgrading rates $(\mathrm{OR}, 1.12 ; P=.03)$ and higher upgrading and/or upstaging rates (OR, 1.15; $P=.01)$ but not for higher upstaging rates $(P>.05)$. Finally, in patients with favorable intermediate risk, marginally higher rates of upgrading, upstaging, or upgrading and/or upstaging were recorded in White and not in Black patients $(-0.7 \%$ to $-1.6 \%)$, and Black race failed to reach independent predictor status in each endpoint.

Taken together, these observations indicate that Black race does not invariably predispose to higher upgrading or upstaging, except for a very modestly increased risk in patients with very low risk but not in those with low or favorable intermediate risk. Consequently, Black patients may be given the same consideration as White patients when AS is an option based on the NCCN 
A

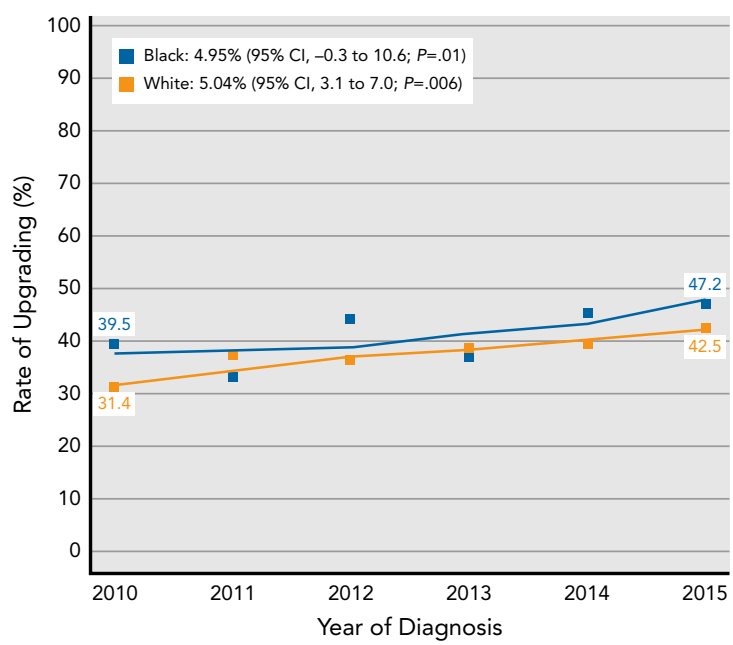

B

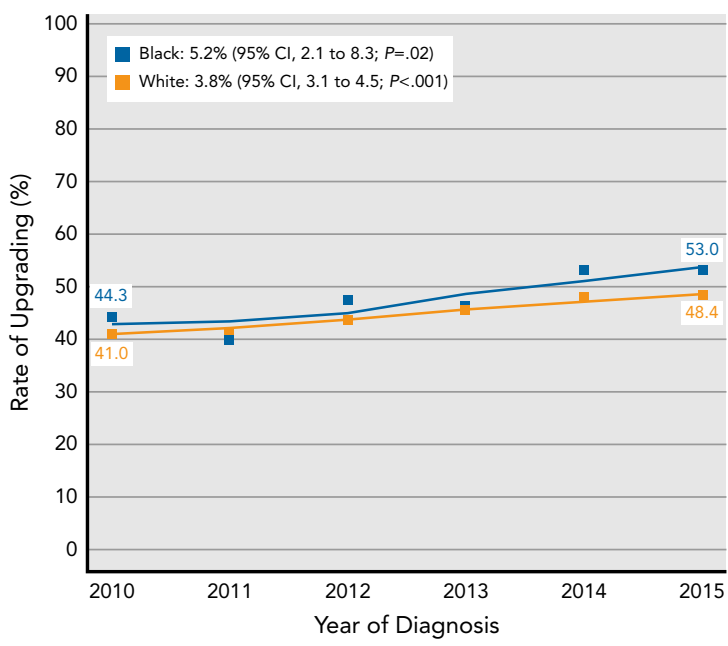

C

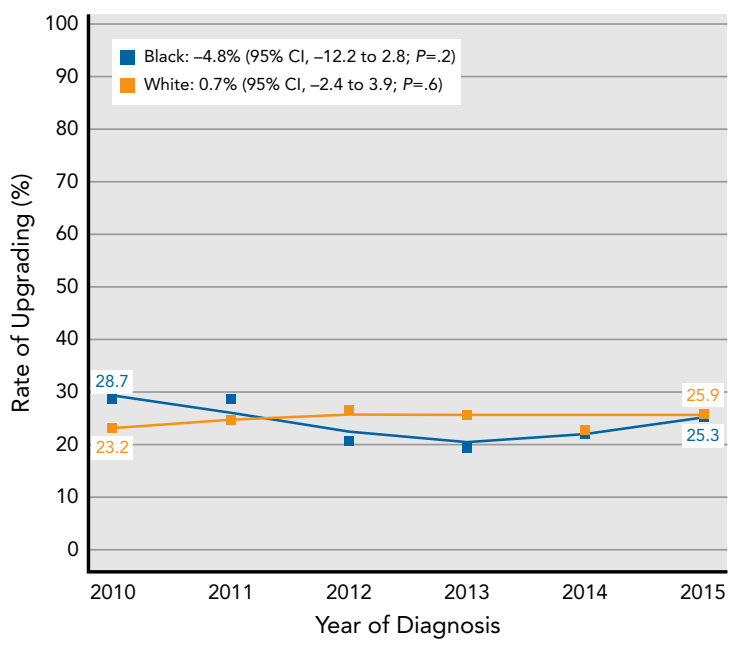

Figure 2. Temporal trends of upgrading rates within the $(A)$ very-lowrisk, (B) low-risk, and (C) favorable intermediate-risk groups.
Guidelines ${ }^{1}$ very-low, low-, or favorable intermediate-risk criteria. The earlier observations validate the original hypothesis about the absence of meaningful differences between Black and White patients with respect to upgrading and/or upstaging. Moreover, these observations agree with previous historical studies in which Black race was not associated with higher misclassification rates. $^{5,6}$ Finally, a recent population-based study showed that Black patients had similar 10-year PCaspecific mortality (PCSM) rates after RP relative to White patients. ${ }^{7}$

Second, temporal trend analyses that focused on upgrading rates over time (2010-2015) in the 3 AS categories showed no meaningful differences in either Black or White patients. In patients with very low risk, upgrading rates increased in Black patients from $39.5 \%$ to $47.2 \%(P=.01)$ and in White patients from $31.4 \%$ to $42.5 \%$ $(P<.006)$. Similarly, in patients with low risk, an increase over time was recorded for Black patients $(44.3 \%-53.0 \%$; $P=.02)$ and in White patients $(41.0 \%-48.4 \% ; P<.001)$. Conversely, in patients with favorable intermediate risk, no differences over time were recorded between Black and White patients ( $P>.05$ for both). Taken together, the magnitude of increase in upgrading was very similar between Black (8\%-9\%) and White patients $(7 \%-11 \%)$. However, the absolute starting point for the upgrading rates was invariably higher in Black compared with White patients. Consequently, the increase in upgrading rates also invariably culminated in higher absolute rates in Black than in White patients. Nonetheless, these differences may be not clinically meaningful in individual patients. However, they may be of importance in the context of clinical trial planning and design.

Third, unexpectedly elevated rates of upgrading were seen in both Black and White patients within all 3 AS categories. Specifically, in patients with very low risk and low risk, upgrading rates ranged from $36.4 \%$ to $46.0 \%$ compared with $24.0 \%$ to $24.7 \%$ in patients with favorable intermediate risk. Consequently, in patients with very low or low risk, upgrading should be expected in more than 1 in 3 individuals. Conversely, in patients with favorable intermediate risk, upgrading should be expected in as many as 1 in 4 individuals. Such misclassification rates in GGG seem elevated, especially in patients with very low and low risk. Although most upgrading in these individuals occurred from GGG 1 to GGG $2(30.3 \%-39.5 \%)$, between $5.7 \%$ and $7.0 \%$ of these patients were upgraded to GGG 3, 4, or 5 . The natural history of GGG 3, 4, or 5 is clearly different from that of GGG 1 . Indeed, Pompe et $\mathrm{al}^{8}$ reported significantly worse 8-year PCSM-free survival rates in patients with RP GGG 3, 4, and 5 (85.8\%-97.4\%) than in those with RP GGG 1 or $2(99.1 \%-99.5 \% ; P<.001)$. However, the ProtecT trial focused on 10-year PCSM-free survival and did not detect important differences between 
the AS and RP arms (98.8\% vs $99.0 \% ; P=.5) .{ }^{9}$ Moreover, a recently published study of a prospective AS cohort $(n=1,818)$ also reported a 15-year PCSM-free survival of 99.9\%. ${ }^{10}$ Therefore, most upstaged and/or upgraded patients will respond to treatment without excess mortality. Nonetheless, upgrading and/or upstaging due to misclassification errors may result in more treatmentintense follow-up and in higher total health expenditures.

Fourth, unlike for upgrading rates that ranged from $24.0 \%$ to $46.0 \%$, upstaging rates in patients with very low, low, and favorable intermediate risk were lower and ranged from $6.9 \%$ to $17.4 \%$. However, upstaged patients exhibited pT3 or higher stages and/or pN1 stage. Therefore, the consequences of upstaging are worse than those of upgrading, even when upgrading results in GGG 3, 4, or 5. It is of interest that most upstaged patients will also be upgraded (37.0\%-80.2\%). Therefore, it can be argued that the seemingly low absolute rates of upstaging have dire consequences on the treated natural history of patients with initially very low, low, or favorable intermediate risk for PCa. Indeed, Gandaglia et $\mathrm{al}^{11}$ reported a 5-year biochemical recurrence-free survival of $79.1 \%$ in patients upgraded to GGG 3, 4, or 5 or those upstaged to pT3+/pN1 compared with $97.0 \%$ in patients upgraded to GGG $2(P<.001)$.

Our work distinguishes itself from previous reports because it relies on more contemporary and detailed AS definitions. Specifically, Katz et $\mathrm{al}^{5}$ relied on the SEER database from 2004 to 2013 and reported no differences in upgrading rates (7.0\% vs $7.0 \%)$ between Black $(n=1,009)$ and White patients $(n=6,346)$. However, in their analyses, PSA and cT stage were not considered. Consequently, they included patients with a PSA level and cT stage outside of the allowed NCCN Guidelines AS scope. Moreover, they focused on patients upgrading to GGG 3, 4, or 5 but not those upgrading to GGG 2. Based on these limitations, it is difficult if not impossible to interpret their results in the context of the NCCN AS recommendations. ${ }^{1}$ Moreover, it is impossible to compare the Katz et $\mathrm{al}^{5}$ findings with institutional databases that strictly adhere to the NCCN AS recommendations. Indeed, important differences distinguish the Katz et al ${ }^{5}$ findings from those of other institutional reports that focused on upgrading and/or upstaging. For example, Faisal et $\mathrm{al}^{12}$ reported on their institutional experience (1992-2013) with patients with very low risk and low risk, including 1,634 Black and 15,993 White patients. In their report, upgrading rates for Black and White patients with very low risk were $29.3 \%$ versus $15.4 \%$, respectively, and $30.8 \%$ versus $24.9 \%$, respectively, for those with low risk. ${ }^{12}$

Taken together, we recorded unexpectedly high rates of misclassification between biopsy and RP tumor grade and stage in AS candidates within this large contemporary cohort of Black and White patients. Consequently, AS definitions may require a recalibration to reduce misclassification rates. Moreover, Black race does not predispose to higher upgrading or upstaging rates, except in a very modest fashion in patients with PCa with very low risk. Finally, our data seem to indicate that Black patients may be given the same consideration as White patients when AS is an option. However, further validations should ideally follow.

Our work has limitations and should be interpreted in the context of its retrospective and population-based design. First, it does not allow adjustment or specific analyses that focus on earlier cancer control endpoints, such as biochemical recurrence rates. Second, no information regarding PSA density and the percentage of cancer per core was available in the SEER database. Moreover, we identified $2.1 \%(\mathrm{n}=101)$ of cTla and $1.8 \%$ $(\mathrm{n}=86)$ of cTlb tumors within patients with very low risk. We analyzed the data after deletion of these cases and arrived at exactly the same results. Therefore, cT1 stage can be considered as Tlc for practical purposes. Third, we could not account for selection biases related to primary treatment assignment to RP. This surgical cohort may be not reflective of all patients with newly diagnosed PCa according to age and comorbidity profile. Fourth, we could not account for the use of additional staging tools, such as MRI. It is possible that the rates of prostate MRI have increased over time and approximate MRI use in prospective trials. However, it is unlikely that the follow-up of included patients in the SEER database approximates any formal and structured AS protocol. ${ }^{1}$ Similar considerations apply to the use of follow-up biopsies that are not recorded in the SEER database. However, these limitations as well as those related to the retrospective nature of the SEER database apply to all other population-based analyses that have been derived from the SEER or other similar large-scale data repositories. ${ }^{4}$

\section{Conclusions}

Rates of misclassification were particularly elevated in patients with very low risk and low risk regardless of Black or white race and ranged from $33.8 \%$ to $48.6 \%$. Recalibration of very-low-risk, low-risk, and, to a lesser extent, favorable intermediate-risk AS criteria may be required. Finally, our data seem to indicate that Black patients may be given the same consideration as White patients when AS is an option.

Submitted December 19, 2019; accepted for publication April 27, 2020.

Author contributions: Content counseling: Stolzenbach, Rosiello, Pecoraro, Palumbo, Luzzago, Deuker, Knipper, Pompe. Statistics: Stolzenbach, Tian. Review: Zorn, Shariat, Chun, Graefen, Saad, Karakiewicz. Supervisor: Karakiewicz. 
Disclosures: The authors have disclosed that they have not received any financial consideration from any person or organization to support the preparation, analysis, results, or discussion of this article.
Correspondence: Lara Franziska Stolzenbach, MD Martini-Klinik Prostate Cancer Center, University Hospital Hamburg-Eppendorf, Martinistraße 52, 20246 Hamburg, Germany. Email: fstolzenbach191@web.de

\section{References}

1. Mohler JL, Higano CS, Pugh TJ, et al. NCCN Clinical Practice Guidelines in Oncology: Prostate Cancer. Version 4.2019. Accessed April 30, 2020. To view the most recent version, visit NCCN.org

2. Epstein JI, Walsh PC, Carmichael M, et al. Pathologic and clinical findings to predict tumor extent of nonpalpable (stage T1c) prostate cancer. JAMA 1994;271:368-374.

3. Bastian PJ, Mangold LA, Epstein JI, et al. Characteristics of insignificant clinical T1c prostate tumors. Cancer 2004;101:2001-2005.

4. National Cancer Institute. Overview of the SEER program. Accessed April 30, 2020. Available at: https://seer.cancer.gov/about/overview.html

5. Katz JE, Chinea FM, Patel VN, et al. Disparities in Hispanic/Latino and nonHispanic black men with low-risk prostate cancer and eligible for active surveillance: a population-based study. Prostate Cancer Prostatic Dis 2018;21:533-538.

6. Leapman MS, Freedland SJ, Aronson WJ, et al. Pathological and biochemical outcomes among African-American and Caucasian men with low risk prostate cancer in the SEARCH Database: implications for active surveillance candidacy. J Urol 2016;196:1408-1414.

7. Dess RT, Hartman HE, Mahal BA, et al. Association of black race with prostate cancer-specific and other-cause mortality. JAMA Oncol 2019;5:975-983.
8. Pompe RS, Davis-Bondarenko $\mathrm{H}$, Zaffuto $\mathrm{E}$, et al. Population-based validation of the 2014 ISUP Gleason grade groups in patients treated with radical prostatectomy, brachytherapy, external beam radiation, or no loca treatment. Prostate 2017;77:686-693.

9. Hamdy FC, Donovan JL, Lane JA, et al. 10-year outcomes after monitoring, surgery, or radiotherapy for localized prostate cancer. $\mathrm{N}$ Engl J Med 2016;375:1415-1424.

10. Tosoian JJ, Mamawala M, Epstein JI, et al. Intermediate and longer-term outcomes from a prospective active-surveillance program for favorable-risk prostate cancer. J Clin Oncol 2015;33 3379-3385

11. Gandaglia G, Ploussard G, Isbarn H, et al. What is the optimal definition of misclassification in patients with very low-risk prostate cancer eligible for active surveillance? Results from a multi-institutional series. Urol Oncol 2015;33:164.e1-9

12. Faisal FA, Sundi D, Cooper JL, et al. Racial disparities in oncologic outcomes after radical prostatectomy: long-term follow-up. Urology 2014 84:1434-1441.

\section{Explore Oncology From Every Angle}

The Hub for Disease-Specific Clinical Information

JNCCN Spotlights: exclusive commentary about current therapies

$>$ Video interviews with experts
$>$ Links to professional and patient resources

$>$ Summaries of news and medical literature

\section{JNCCN360.org}

\&IARBORSIDE

The nexus of knowledge
National NCCN Comprehensive NCCN $\begin{aligned} & \text { Cancer } \\ & \text { Network }\end{aligned}$ 
Supplemental online content for:

\section{Prostate Cancer Grade and Stage Misclassification in Active Surveillance Candidates: Black Versus White Patients}

Lara Franziska Stolzenbach, MD; Giuseppe Rosiello, MD; Angela Pecoraro, MD; Carlotta Palumbo, MD; Stefano Luzzago, MD; Marina Deuker, MD; Zhe Tian, MD; Anne-Sophie Knipper, MD; Raisa Pompe, MD; Kevin C. Zorn, MD; Shahrokh F. Shariat, MD; Felix K.H. Chun, MD; Markus Graefen, MD; Fred Saad, MD; and Pierre I. Karakiewicz, MD

J Natl Compr Canc Netw 2020;18(11):1492-1499

eTable 1: Effect of Black Race on Upgrading and/or Upstaging Within the Very-Low-Risk Group eTable 2: Effect of Black Race on Upgrading and/or Upstaging Within the Low-Risk Group eTable 3: Effect of Black Race on Upgrading and/or Upstaging Within the Favorable Intermediate-Risk Group 


\begin{tabular}{|c|c|c|c|c|c|c|}
\hline Variable & $\begin{array}{c}\text { Multivariable OR } \\
\quad(95 \% \mathrm{Cl})\end{array}$ & $P$ Value & $\begin{array}{c}\text { Multivariable OR } \\
(95 \% \mathrm{Cl})\end{array}$ & $P$ Value & $\begin{array}{c}\text { Multivariable OR } \\
(95 \% \mathrm{Cl})\end{array}$ & $P$ Value \\
\hline \multicolumn{7}{|l|}{ Race } \\
\hline White & Ref & & Ref & & Ref & \\
\hline Age at diagnosis & $1.05(1.01-1.09)$ & .006 & $1.02(1.01-1.04)$ & $<.001$ & $1.02(1.01-1.03)$ & $<.001$ \\
\hline PSA & $1.01(1.00-1.02)$ & .001 & $1.03(1.00-1.57)$ & .04 & $1.01(1.00-1.02)$ & .006 \\
\hline Number of cores & $0.96(0.93-0.97)$ & $<.001$ & $0.98(0.94-1.01)$ & .4 & $0.9(0.94-1.02)$ & .4 \\
\hline Number of positive cores & $1.40(1.23-1.58)$ & $<.001$ & $1.26(1.01-1.57)$ & .04 & $1.04(0.68-1.59)$ & .8 \\
\hline
\end{tabular}

All models adjusted for age at diagnosis, preoperative PSA level, number of positive cores, and number of cores. Abbreviations: OR, odds ratio; PSA, prostate-specific antigen.

\section{eTable 2. Effect of Black Race on Upgrading and/or Upstaging Within the Low-Risk Group}

\begin{tabular}{|c|c|c|c|c|c|c|}
\hline \multirow[b]{2}{*}{ Variable } & \multicolumn{2}{|c|}{ Upgrading } & \multicolumn{2}{|c|}{ Upstaging } & \multicolumn{2}{|c|}{ Upgrading and/or Upstaging } \\
\hline & $\begin{array}{c}\text { Multivariable OR } \\
\quad(95 \% \mathrm{Cl})\end{array}$ & $P$ Value & $\begin{array}{c}\text { Multivariable OR } \\
\quad(95 \% \mathrm{Cl})\end{array}$ & $P$ Value & $\begin{array}{c}\text { Multivariable OR } \\
(95 \% \mathrm{CI})\end{array}$ & $P$ Value \\
\hline White & Ref & & Ref & & Ref & \\
\hline Black & $1.12(1.00-1.25)$ & .03 & $0.85(0.70-1.02)$ & .09 & $1.15(1.03-1.28)$ & .01 \\
\hline Number of cores & $0.97(0.95-0.98)$ & $<.001$ & $0.96(0.93-0.99)$ & .01 & $0.97(0.94-0.99)$ & $<.001$ \\
\hline Number of positive cores & $1.40(1.23-1.58)$ & $<.001$ & $1.26(1.01-1.57)$ & .04 & $1.12(1.07-1.17)$ & $<.001$ \\
\hline Core ratio & $1.08(0.69-1.67)$ & .7 & $1.09(0.53-2.19)$ & .8 & $0.87(0.50-1.53)$ & .6 \\
\hline
\end{tabular}

All models adjusted for age at diagnosis, preoperative PSA level, number of positive cores, and number of cores. Abbreviations: OR, odds ratio; PSA, prostate-specific antigen.

\begin{tabular}{|c|c|c|c|c|c|c|}
\hline \multirow[b]{2}{*}{ Variable } & \multicolumn{2}{|c|}{ Upgrading } & \multicolumn{2}{|c|}{ Upstaging } & \multicolumn{2}{|c|}{ Upgrading and/or Upstaging } \\
\hline & $\begin{array}{c}\text { Multivariable OR } \\
(95 \% \mathrm{Cl})\end{array}$ & $P$ Value & $\begin{array}{c}\text { Multivariable OR } \\
(95 \% \mathrm{Cl})\end{array}$ & $P$ Value & $\begin{array}{c}\text { Multivariable OR } \\
(95 \% \mathrm{Cl})\end{array}$ & $P$ Value \\
\hline \multicolumn{7}{|l|}{ Race } \\
\hline Age at diagnosis & $1.02(1.01-1.02)$ & $<.001$ & $1.02(1.01-1.04)$ & $<.001$ & $1.01(1.02-1.03)$ & $<.001$ \\
\hline PSA & $1.13(1.11-1.14)$ & $<.001$ & $1.07(1.06-1.09)$ & $<.001$ & $1.11(1.10-1.13)$ & $<.001$ \\
\hline Number of cores & $1.01(0.98-1.03)$ & .6 & $1.01(0.97-1.03)$ & .6 & $1.01(0.98-1.02)$ & .6 \\
\hline Number of positive cores & $0.9(0.82-1.01)$ & .07 & $0.99(0.89-1.09)$ & .8 & $0.94(0.86-1.04)$ & .2 \\
\hline
\end{tabular}

All models adjusted for age at diagnosis, preoperative PSA level, number of positive cores, and number of cores. Abbreviations: OR, odds ratio; PSA, prostate-specific antigen. 